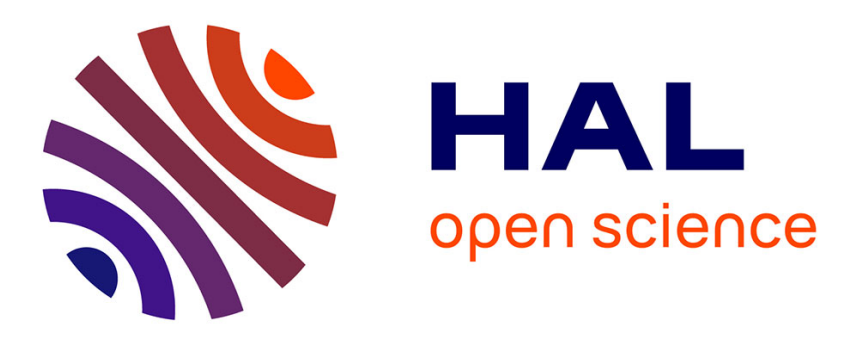

\title{
Experimental Evidence of the Néel-Brown Model of Magnetization Reversal
}

\author{
W. Wernsdorfer, E Bonet Orozco, Klaus Hasselbach, A. Benoît, B. Barbara, \\ N. Demoncy, A. Loiseau, H. Pascard, D. Mailly
}

\section{- To cite this version:}

W. Wernsdorfer, E Bonet Orozco, Klaus Hasselbach, A. Benoît, B. Barbara, et al.. Experimental Evidence of the Néel-Brown Model of Magnetization Reversal. Physical Review Letters, 1997, 78 (9), pp.1791. hal-01659965

\section{HAL Id: hal-01659965 https://hal.science/hal-01659965}

Submitted on 9 Dec 2017

HAL is a multi-disciplinary open access archive for the deposit and dissemination of scientific research documents, whether they are published or not. The documents may come from teaching and research institutions in France or abroad, or from public or private research centers.
L'archive ouverte pluridisciplinaire HAL, est destinée au dépôt et à la diffusion de documents scientifiques de niveau recherche, publiés ou non, émanant des établissements d'enseignement et de recherche français ou étrangers, des laboratoires publics ou privés. 


\title{
Experimental Evidence of the Néel-Brown Model of Magnetization Reversal
}

\author{
W. Wernsdorfer, ${ }^{1,2}$ E. Bonet Orozco, ${ }^{1,2}$ K. Hasselbach, ${ }^{1}$ A. Benoit, ${ }^{1}$ B. Barbara, ${ }^{2}$ N. Demoncy, ${ }^{3,4}$ A. Loiseau ${ }^{4}$ \\ H. Pascard, ${ }^{3}$ and D. Mailly ${ }^{5}$ \\ ${ }^{1}$ CRTBT-CNRS, BP166, 38042 Grenoble, France \\ ${ }^{2}$ LL Néel-CNRS, BP166, 38042 Grenoble, France \\ ${ }^{3}$ SESI-CEA/CNRS, École Polytechnique, 91128 Palaiseau, France \\ ${ }^{4}$ OM-ONERA, BP 72, 92322 Chatillon, France \\ ${ }^{5}$ LMM-CNRS, 196 Av. H. Ravera, 92220 Bagneux, France
}

(Received 22 August 1996)

\begin{abstract}
Presented are the first magnetization measurements of individual ferromagnetic nanoparticles (15$30 \mathrm{~nm})$ at very low temperatures $(0.1-6 \mathrm{~K})$. The angular dependence of the hysteresis loop evidenced the single domain character of the particles. Waiting time, switching field, and telegraph noise measurements showed for the first time that the magnetization reversal of a well prepared ferromagnetic nanoparticle can be described by thermal activation over a single-energy barrier as originally proposed by Néel and Brown. The "activation volume" estimated by these measurements was close to the particle volume. [S0031-9007(97)02465-4]
\end{abstract}

PACS numbers: 75.40.Gb, 75.50.Cc, 75.50.Tt

The thermal fluctuations of the magnetic moment of a single-domain ferromagnetic particle and its decay toward thermal equilibrium was introduced by Néel [1] and further developed by Brown [2]. Both assumed uniform magnetization and uniaxial anisotropy in order to derive a single relaxation time. The main difference between their results lies in the pre-exponential factor [3] which remains an unsolved problem [4]. This prefactor depends on several parameters as, e.g., damping and temperature [5]. For simplicity, experimentalists supposed a constant prefactor, and this assumption became known as the NéelBrown model. This model is widely used in magnetism, particularly in order to describe the time dependence of the magnetization of collections of particles, thin films, and bulk materials. Therefore, a good understanding of the problem of magnetization reversal in these complex systems requires an understanding of magnetization reversal processes in a single magnetic particle. Until recently, almost all experimental studies were limited to samples consisting of billions of presumably identical particles. Most of the single-particle properties were hidden behind some distribution functions of particle size, shape etc. Only in some few cases, measurements performed on individual single-domain particles have been reported [6], but they lacked the ability to provide quantitative information. With the recent arrival of near field microscopy, (magnetic force microscopy) and nanolithography, experimental studies of magnetization reversal in individual particles became possible, e.g., [7,8]. Until now, all the reported measurements, performed on individual particles, were not consistent with the Néel-Brown model of thermally assisted magnetization reversal over a simple potential barrier. This disagreement was attributed to the fact that real samples contain defects, ends, and surfaces which could play an important, if not dominant, role in the physics of magnetization reversal. It was suggested that the dynamics of reversal occurs via a complex path in configuration space, and that a new theoretical approach is required to provide a correct description of thermally activated magnetization reversal even in single-domain ferromagnetic particles [9]. Similar conclusions were drawn from numerical simulations of the magnetization reversal [10].

It is the purpose of this Letter to show for the first time that the magnetization reversal of a well prepared ferromagnetic nanoparticle can be described by thermal activation over a single-energy barrier as originally proposed by Néel and Brown [1,2].

For ferromagnetic particles and at zero field, the energy barrier between the two states of opposite magnetization is much too high to observe magnetization reversal. However, the barrier can be lowered by, e.g., applying a magnetic field in the opposite direction of the particle's magnetization. When the applied field is close to the switching field at zero temperature $H_{\mathrm{sw}}^{0}$, thermal fluctuations are sufficient enough to drive the system to overcome the barrier. A simple analytical approximation for the field dependence of the energy barrier $E(H)$ is

$$
E(H)=E_{0}\left(1-H / H_{\mathrm{sw}}^{0}\right)^{\alpha}=E_{0} \varepsilon^{\alpha},
$$

where $E_{0}$ is an extrapolation of the energy barrier at zero field, and $\varepsilon$ is defined as a reduced field difference value. It can be shown that the exponent $\alpha$ is in general equal to $1.5[11,12]$. The probability that the magnetization has not switched after a time $t$ is given by

$$
P(t)=e^{-t / \tau},
$$

where $\tau$ (inverse of the switching rate) can be expressed by an Arrhenius law of the form

$$
\tau(T, H)=\tau_{0} \exp (E(H) / k T),
$$


where $\tau_{0}$ (inverse of the attempt frequency) depends on several parameters as, e.g., the damping and the temperature [5]. For simplicity, we supposed a constant prefactor $\tau_{0}$.

For the experiments, it is often more convenient to study the magnetization reversal by ramping the applied field at a given rate and measuring the field value as soon as the particle's magnetization switches. In this case, thermal activation leads to a distribution of switching fields initially evaluated by Kurkijärvi [13]. The mean switching field $H_{\text {sw }}$ is given by

$$
H_{\mathrm{sw}} \cong H_{0}\left\{1-\left[\frac{k T}{E_{0}} \ln \left(\frac{c T}{v \varepsilon^{\alpha-1}}\right)\right]^{1 / \alpha}\right\},
$$

where $c=k H_{0} /\left(\tau_{0} \alpha E_{0}\right)$ and $v$ is the field sweeping rate. The width of the switching field distribution is given by

$$
\sigma \cong H_{0} \frac{1}{\alpha}\left(\frac{k T}{E_{0}}\right)^{1 / \alpha}\left[\ln \left(\frac{c T}{v \varepsilon^{\alpha-1}}\right)\right]^{(1-\alpha) / \alpha} .
$$

In order to test the validity of the Néel-Brown model, we studied nanosized $\mathrm{Ni}$, Co, and Dy particles (15$30 \mathrm{~nm}$ ) synthesized by arc discharge [14]. According to transmission electron microscope observations, these particles are single crystalline with a surface roughness of about two atomic layers, and they are encapsulated in either carbon graphitic shells or amorphous carbon protecting them very efficiently against oxidation [14].

We used a planar Nb micro-bridge-dc SQUID (of 1 to $2 \mu \mathrm{m}$ in diameter) on which we placed a ferromagnetic particle. The SQUID detected the flux through its loop produced by the sample's magnetization. Because of the close proximity between sample and SQUID we had a very efficient and direct flux coupling. In this configuration, we could detect magnetization reversals corresponding to $10^{4} \mu_{B}$ [15]. In order to place a nanoparticle on the SQUID detector, we dispersed the particles in ethanol by ultrasonication. Then we placed a drop of this liquid on a chip of about a hundred SQUIDs. When the drop was dry the nanoparticles stuck on the chip due to van der Waals forces. Only in the case when a nanoparticle fell on a microbridge of the SQUID loop, the flux coupling between SQUID loop and particle was strong enough to be detected. After the magnetization measurements, we finally determined the position and size of the nanoparticles by scanning electron microscopy (SEM).

In this Letter, we focus on an ellipsoidal Co particle with a fcc crystalline structure and a diameter of $25 \pm$ $5 \mathrm{~nm}$ (see Fig. 1). In order to study the domain structure and the reversal mode of this nanoparticle, we measured the angular dependence of hysteresis loops. The magnetic field was applied in the plane of the SQUID. The hysteresis loop was reversible up to the switching field, the external field value where the magnetization of the particle flips in the opposite direction. This switching was in all cases faster than out time resolution of $100 \mu \mathrm{s}$. Figure 1 shows the angular dependence of the switching field. As the easy axis of magnetization of the particle

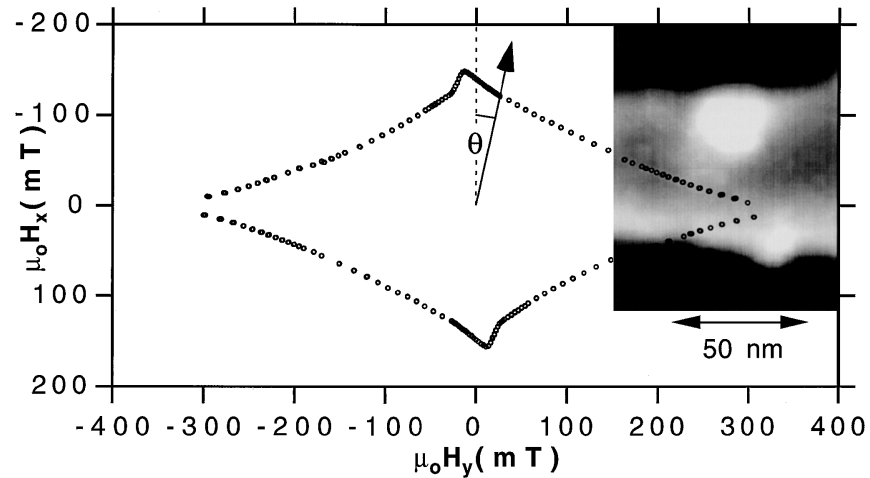

FIG. 1. Angular dependence of the switching field of an ellipsoidal Co nanoparticle $(25 \pm 5 \mathrm{~nm}$ in diameter) deposed on a microbridge. The arrow indicates the direction of the applied field chosen for the waiting time measurements in Fig. 2 and for the switching field measurements in Figs. 35. For the telegraph noise measurements in Fig. 6, a high field in the $H_{y}$ direction was applied. Inset: SEM photo of the Co particle on the microbridge (white spot).

did not lie in the SQUID plane, we chose the following orientation of the field: $H_{x}$ and $H_{y}$ are in the SQUID plane so that the $H_{x}$ direction is parallel to the in-the-SQUIDplane-projected easy axis; i.e., the $H_{y}$ direction of the applied field is perpendicular to the easy axis. The angle $\theta$ is measured between the $H_{x}$ direction and the applied in-plane field (see Fig. 1). By applying an additional constant field perpendicular to the SQUID plane, we found that the easy axis of magnetization of the particle was out of the SQUID plane by about $20^{\circ}$. The angular dependence of $H_{\text {sw }}$ compared favorably with the model of magnetization reversal by uniform rotation in the presence of two anisotropy axes [16]. A detailed discussion of these results is presented elsewhere [17].

After having characterized the static magnetic properties, we studied the stochastic character of the switching field by waiting time, switching field, and telegraph noise measurements. Via the waiting time measurement we had direct access to the switching probability. At a given temperature, the magnetic field was increased to a waiting field $H_{w}$ which was close to the switching field. Then, we

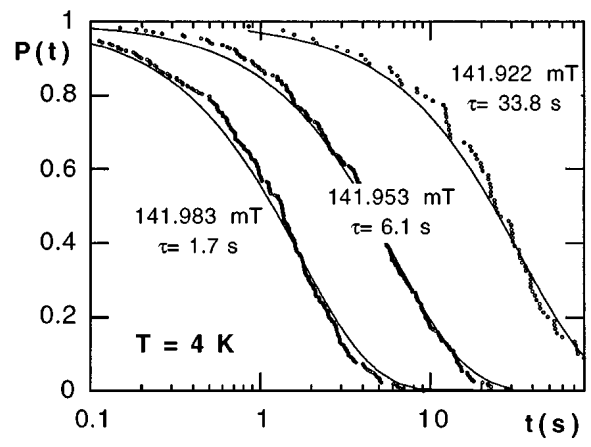

FIG. 2. Probability of not switching of magnetization as a function of the time at different applied fields at $4 \mathrm{~K}$ and for $\theta \approx 12^{\circ}$. Full lines are fits to the data with an exponential [Eq. (2).] 


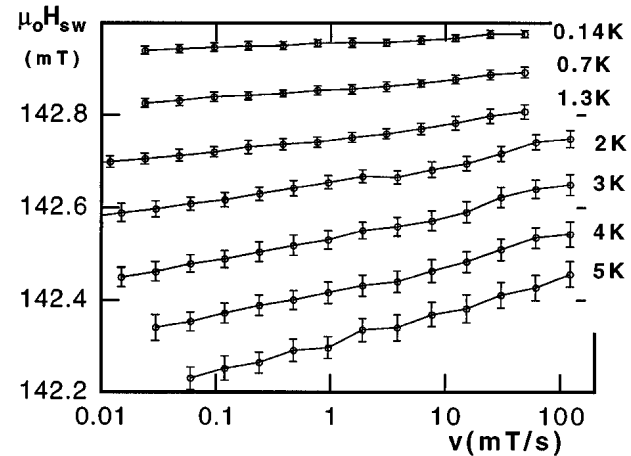

FIG. 3. Thermal and field sweeping rate dependence of the mean switching field for $\theta \approx 12^{\circ}$. The widths $\sigma$ of the switching field distributions are indicated by vertical bars.

measured the elapsed time until the magnetization switched. This process was repeated several hundred times, in order to obtain a waiting time histogram. The integral of this histogram gave us the switching probability. All our measurements showed that the probability of switching is given by an exponential in agreement with Eq. (2). Figure 2 shows examples of the measured probability of switching at $4 \mathrm{~K}$. Moreover, we found that the field and temperature dependence of $\tau$ followed Eq. (3) [17].

Concerning the switching field measurements, the applied field was ramped at a given rate and the field value was stored as soon as the sample magnetization switched. Then the field ramp was reversed and the process was repeated. After several hundred cycles, switching field histograms were established, allowing us to define the mean switching field $H_{\mathrm{sw}}$ and widths $\sigma$. At temperatures between 0.1 and $6 \mathrm{~K}$, we measured $H_{\mathrm{sw}}$ for field sweeping rates between 0.01 and $120 \mathrm{mT} / \mathrm{s}$. As expected for a thermally activated process, the mean switching field increased with decreasing temperature and increasing field sweeping rate. Furthermore, all our measurements showed an almost logarithimic dependence of $H_{\text {sw }}$ on the field sweeping rate (Fig. 3). The validity of Eq. (4) was tested by plotting the mean switching field values as a function of $\left[T \ln \left(c T / v \varepsilon^{\alpha-1}\right)\right]^{1 / \alpha}$. If the underlying model is sufficient, all points should collapse onto one straight line by choosing the proper values for the constants $c$ and $\alpha$. We found that the data of $H_{\mathrm{sw}}(T, v)$ fell on a master curve provided $c \approx 10^{5} \mathrm{mT} / \mathrm{Ks}$ (Fig. 4 ), i.e., $\tau_{0} \approx 4 \times 10^{-9} \mathrm{~s}$. For the choice of $\alpha=1.5 \pm 0.05$, the master curve is a straight line. The slope and the intercept give the value of the $E_{0}=214000 \mathrm{~K}$ and $H_{\mathrm{sw}}^{0}=143.1 \mathrm{mT}$ corresponding to those found in the switching time experiments. The inset of Fig. 4 shows a small deviation from the master curve at temperatures lower than $0.3 \mathrm{~K}$ which should be confirmed by experiments at lower temperatures. Using the constants found by this scaling plot, Eq. (5) describes well the measured temperature dependence of the width of the switching field distribution $\sigma$ (Fig. 5).

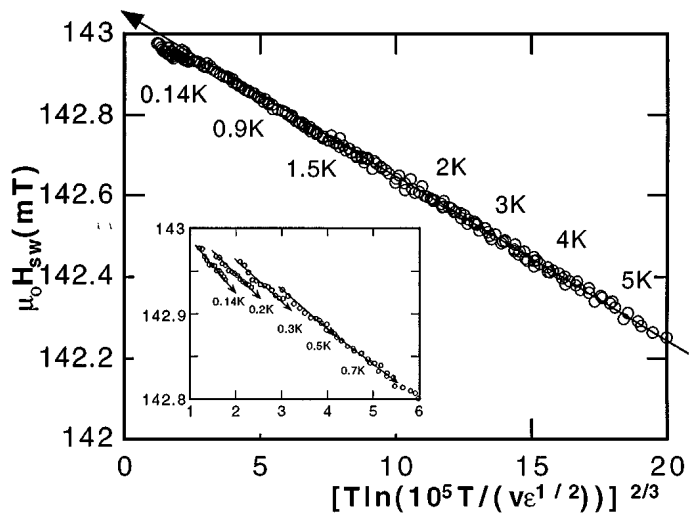

FIG. 4. Scaling plot of the mean switching fields $H_{\mathrm{sw}}$ for field sweeping rates between $0.01-120 \mathrm{mT} / \mathrm{s}$, temperatures between $0.14-5 \mathrm{~K}$ and for $\theta \approx 12^{\circ}$. Inset; Zoom for lower temperatures.

From the waiting time and the switching field measurements we could determine the energy barrier $E_{0}$ which can be approximately converted to a thermally "activated volume" by using $V=E_{0} /\left(\mu_{0} M_{s} H_{\mathrm{sw}}\right)$. We found $V \approx(25 \mathrm{~nm})^{3}$ which is very close to the particle volume estimated by SEM. This agreement is another confirmation of a magnetization reversal by uniform rotation. We found similar results for measurements at angles $\theta$ between $0^{\circ}$ and $80^{\circ}$.

For the telegraph noise measurements [18], we applied a constant field in the $H_{y}$ direction in order to reduce the height of the energy barrier. When the energy barrier is sufficiently small, the magnetization of the particle fluctuates between two orientations which are close to the $H_{y}$ direction (Fig. 6). The time spent in each state followed an exponential distribution as given by Eq. (2). The switching was thermally activated and the mean switching time $\tau$ followed Eq. (3) with $\tau_{0}$ of the order of $10^{-9} \mathrm{~s}$, which is several orders of magnitude smaller than $\tau_{0}$ measured for small ErAs clusters by magnetoresistance measurements [19].

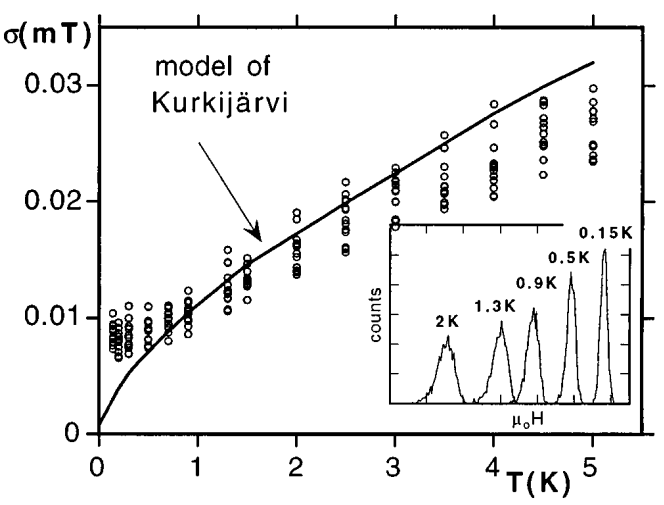

FIG. 5. Temperature dependence of the width of the switching field distribution $\sigma$ for $\theta \approx 12^{\circ}$ and $\mu_{0} d H / d t=0.01-120 \mathrm{mTs}$. Line: prediction of Kurkijärvi [13] for $\mu_{0} d H / d t=1 \mathrm{mT} / \mathrm{s}$. Inset: Switching fields distributions for several temperatures and $\mu_{0} d H / d t=60 \mathrm{mT} / \mathrm{s}$. 


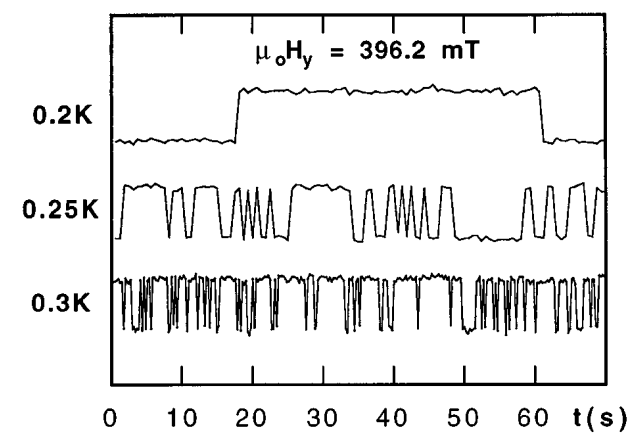

FIG. 6. Telegraph noise measurements for three temperatures and $\mu_{0} H_{y}=396.2 \mathrm{mT}$.

We performed similar measurements on several nanosized $\mathrm{Ni}, \mathrm{Co}$, and Dy particles and found always a good agreement with the Néel-Brown model. However, we systematically observed a disagreement with the NéelBrown model for particles with antiferromagnetic components (due to, e.g., oxidation) or ferrimagnetic materials. In these cases, the probability of not switching was flatter than exponential at low temperature (typically $T<1 \mathrm{~K}$ ) and steeper at higher temperatures [8,9]. Furthermore, the width of the switching field distribution increased for lower temperatures. We believe that the magnetization reversal of these particles is influenced by spin frustration of noncompensated spins at the interface between the ferromagnetic core and the antiferromagnetic surface layers or at the surface of a ferrimagnetic particle. This spin frustration could differ slightly from one hysteresis loop to another, thus producing slightly different energy barriers. This effect is less important at higher temperature when the thermal energy is much larger than the energy barrier variations, whereas at low temperatures this effect may flatten the probability of not switching and increase the width of the switching field distribution. Furthermore, magnetization reversal may be influenced by a reduction of the spin frustration, hence by a relaxation of the energy barrier. This relaxation is thermally activated, i.e., slower at lower temperatures. This effect may be at the origin of the probability of not switching being steeper than exponential at higher temperatures. Similar ideas were discussed in Ref. [8] and [20].

In conclusion, we studied the magnetization switching of individual ferromagnetic particles and observed for the first time the Arrhenius behavior of the switching rate in the temperature range between 0.2 and $6 \mathrm{~K}$. This study has shown the ability of studying the intrinsic magnetization behavior of a single nanoparticle. Our ultimate goal is to study the field and temperature dependence of the prefactor $\tau_{0}$ of Eq. (3). The quality of the particles studied makes feasible the search for a possible macroscopic quantum tunneling of the magnetization [21].
We acknowledge the contributions of B. Pannetier and T. Crozes (CRTBT-CNRS), L. Gunther and the help of D. Boivin (OM-ONERA) for the SEM observations.

[1] L. Néel, Ann. Geophys. 5, 99 (1949).

[2] W.F. Brown, Phys. Rev. 130, 1677 (1963).

[3] D. H. Jones and K. K. P. Srivastava, J. Magn. Magn. Mater. 78, 320 (1989).

[4] W. T. Coffey et al., Phys. Rev. B 52, 15951 (1995).

[5] I. Klik and L. Gunther, J. Stat. Phys. 60, 473 (1990); P. Hänggi, P. Talkner, and M. Borkovec, Rev. Mod. Phys. 62, 251 (1990).

[6] A. H. Morrish and S.P. Yu, Phys. Rev. 102, 670 (1956); J. E. Knowles, IEEE Trans. Magn. 17, 3008 (1981).

[7] M. Lederman, G. A. Gibson, and S. Schultz, J. Appl. Phys. 73, 6961 (1993); T. Chang and J. G. Chu, J. Appl. Phys. 75, 5553 (1994).

[8] W. Wernsdorfer et al., J. Magn. Magn. Mater. 145, 33 (1995); 151, 38 (1995); Phys. Rev. B 53, 3341 (1996); Phys. Rev. Lett. 77, 1873 (1996).

[9] M. Ledermann, S. Schultz, and M. Ozaki, Phys. Rev. Lett. 73, 1986 (1994); J. Appl. Phys. 75, 6217 (1994); D. D. Awschalom and D. P. DiVincenzo, Phys. Today 48, No. 4, 43 (1995).

[10] For example H. L. Richards et al., J. Magn. Magn. Mater. 150, 37 (1995); J. Appl. Phys. 79, 5749 (1996); J. M. Gonzalez et al., Phys. Rev. B 52, 16034 (1995); J. Appl. Phys. 79, 6479 (1996); D. Garcia-Pablos et al., J. Appl. Phys. 79, 6021 (1996).

[11] R. H. Victora, Phys. Rev. Lett. 63, 457 (1989).

[12] Using the Stoner-Wohlfarth analytical expressions of the energy $E(H)$, we can numerically show that $\alpha$ is near 1.5 and increases up to a value of 2 if the applied field forms an angle smaller than a few degrees with the easy axis [W. Wernsdorfer, thesis, Univ. J. Fourier, Grenoble, 1996].

[13] J. Kurkijärvi, Phys. Rev. B 6, 832 (1972); L. Gunther and B. Barbara, Phys. Rev. B 49, 3926 (1994); A. Garg, Phys. Rev. B 51, 15592 (1995).

[14] C. Guerret-Piécourt et al., Nature (London) 372, 761 (1994).

[15] W. Wernsdorfer et al., J. Appl. Phys. 78, 7192 (1995).

[16] Ching-Ray Chang and D. R. Fredkin, J. Appl. Phys. 63, 3435 (1988); Paul L. Fulmek and Hans Hauser, J. Appl. Phys. 76, 6561 (1994).

[17] W. Wernsdorfer et al., (to be published).

[18] P. M. Campbell et al., Phys. Rev. Lett. 67, 1330 (1991).

[19] F. Coppinger et al., Phys. Rev. Lett. 75, 3513 (1995).

[20] R. H. Kodama et al., Phys. Rev. Lett. 77, 394 (1996).

[21] A. J. Leggett et al., Rev. Mod. Phys. 59, 1 (1987); E. M. Chudnovsky and L. Gunther, Phys. Rev. Lett. 60, 661 (1988); M.-C. Miguel and E. M. Chudnovsky, Phys. Rev. B 54, 389 (1996). 